\title{
Flavor landscape of 10D SYM theory with magnetized extra dimensions
}

\author{
Hiroyuki Abe, ${ }^{a}$ Tatsuo Kobayashi, ${ }^{b}$ Hiroshi Ohki, ${ }^{c}$ Keigo Sumita ${ }^{a}$ and \\ Yoshiyuki Tatsuta ${ }^{a}$ \\ ${ }^{a}$ Department of Physics, Waseda University, \\ Tokyo 169-8555, Japan \\ ${ }^{b}$ Department of Physics, Kyoto University, \\ Kyoto 606-8502, Japan \\ ${ }^{c}$ Kobayashi-Maskawa Institute for the Origin of Particles and the Universe (KMI), \\ Nagoya University, Nagoya 464-8602, Japan \\ E-mail: abe@waseda.jp, kobayash@gauge.scphys.kyoto-u.ac.jp, \\ ohki@kmi.nagoya-u.ac.jp, k.sumita@moegi.waseda.jp, \\ y_tatsuta@akane.waseda.jp
}

ABSTRACT: We study the flavor landscape of the visible sector of particle physics models based on a ten-dimensional super Yang-Mills theory compactified on magnetized tori preserving four-dimensional $\mathcal{N}=1$ supersymmetry. Recently, we constructed a semirealistic model which contains the minimal supersymmetric standard model (MSSM) using an ansatz of magnetic fluxes and orbifolding projections. However, we can consider more various configurations of magnetic fluxes and orbifolding projections preserving fourdimensional $\mathcal{N}=1$ supersymmetry. We research systematically such possibilities for leading to MSSM-like models and study their phenomenological aspects.

Keywords: Compactification and String Models, Field Theories in Higher Dimensions, Supersymmetric Effective Theories

ARXIV EPRINT: 1307.1831 


\section{Contents}

1 Introduction 1

2 The 10D SYM theory in $\mathcal{N}=1$ superspace 3

3 Magnetic fluxes and zero-modes 4

3.1 Factorizable flux Ansatz 6

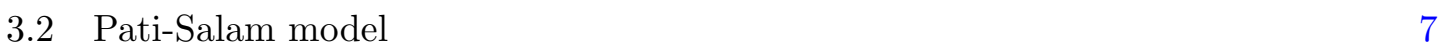

$3.3 Z_{2}$ projection $\quad 9$

4 Nonfactorizable fluxes $\quad 11$

4.1 Nonfactorizable flux Ansatz 13

4.2 Pati-Salam model 14

$\begin{array}{lll}4.3 & \text { Minimal models } & 15\end{array}$

4.4 Nonfactorizable flux models on $T^{6} / Z_{2}$ orbifolds 20

5 Conclusions and discussions $\quad 20$

A Exotic modes with the pattern1 fluxes $\quad 22$

B Yukawa matrices in a one-pair Higgs model 23

\section{Introduction}

The standard model (SM) of elementary particles is the quite successful theory being consistent with all the experimental data so far. However, that is incomplete in several respects. One of important mysteries is the complicated flavor structure. That is, the SM has the three generations of quarks and leptons with hierarchical masses and mixing angles. There should be some underlying mechanisms to understand the flavor structure from the theoretical point of view.

It is known that extra dimensional space can lead rich flavor structures. Especially, simple toroidal compactifications with magnetic fluxes induce the $4 \mathrm{D}$ chiral spectra with hierarchical generation structures in higher-dimensional supersymmetric Yang-Mills (SYM) theories as well as superstring theories [1-5]. ${ }^{1}$ Then, the higher-dimensional supersymmetry (SUSY), such as $\mathcal{N}=4$ in terms of supercharges in $4 \mathrm{D}$ spacetime, is broken by magnetic fluxes down to $4 \mathrm{D} \mathcal{N}=0,1$ or 2 depending on the configuration of fluxes. The number of the chiral zero-modes is determined by the magnitude of magnetic fluxes, and the chiral zero-modes are localized at different points in magnetized extra dimensions. The overlap

\footnotetext{
${ }^{1}$ See for a review [6].
} 
integrals of the localized wavefunctions yield hierarchical couplings in the 4D effective theory of these zero-modes. Actually, that could explain observed hierarchical masses and mixing angles of the quarks and the leptons [7]. Furthermore, even higher-order couplings can also be computed as the overlap integrals of wavefunctions [8]. A theoretically attractive point here is that many peculiar properties of the SM could be determined by the magnetic fluxes, which are the $4 \mathrm{D}$ chirality, the number of generations, the flavor symmetries [9-13] and potentially hierarchical Yukawa couplings.

In our previous works $[14,15]$, we have presented $4 \mathrm{D} \mathcal{N}=1$ superfield description of 10D SYM theories compactified on magnetized tori preserving the $\mathcal{N}=1$ SUSY, and derived $4 \mathrm{D}$ effective action for massless zero-modes written in the $\mathcal{N}=1$ superspace. It makes easy to construct models and analyze them. Thanks to that, we have constructed a three-generation model of quark and lepton chiral superfields based on a toroidal compactification of the 10D SYM theory with certain magnetic fluxes in extra dimensions preserving a $4 \mathrm{D} \mathcal{N}=1$ SUSY. The low-energy effective theory contains the minimal supersymmetric standard model (MSSM) particle contents, where the three generation structure of chiral matter fields originates from magnetic fluxes, and it gives a semi-realistic pattern of hierarchical masses and mixing angles of the quarks and the leptons [15]. Furthermore, we estimated the size of SUSY flavor violations.

One typical model has been studied in our previous works. However, there may be other possibilities for particle physics models based on a 10D SYM theory compactified on three factorizable tori $T^{2} \times T^{2} \times T^{2}$ where magnetic fluxes are present in the YM sector. In this paper, we search systematically such possibilities to obtain models which contain the MSSM and study the flavor landscape of 10D magnetized SYM theories, requiring full rank Yukawa matrices among three generations of quarks and leptons. In this work, we are focusing on only the MSSM sector, so we do not study whole consistency of the system, e.g. anomaly, as well as our previous works. We include possibilities of nonfactorizable magnetic fluxes $[5,16,17]$ (see also [18].) and also consider the $T^{6} / Z_{2}$ orbifold with magnetic fluxes as the geometrical background [19]. ${ }^{2,3}$

The sections are organized as follows. In section 2, we briefly review the superfield description of the 10D SYM theory based on ref. [14], which facilitates the systematic introduction of magnetic fluxes in extra dimensions preserving the $\mathcal{N}=1$ SUSY. Then, we introduce (factorizable) magnetic fluxes and search systematically their proper configurations to construct models that contain the spectrum of the MSSM with and without orbifold projection in section 3. In section 4, we consider magnetic fluxes which cross over two tori. It gives possibilities of more various model building. Including such nonfactorizable fluxes, we carry out a systematic search with and without orbifold projection. Section 5 is devoted to conclusions and discussions. In appendix $\mathrm{A}$, we show one $Z_{2}$ orbifold model, where most of exotic zero-modes are projected out. In appendix B, we show explicitly Yukawa matrices in one type1 model shown in section 4.3.

\footnotetext{
${ }^{2}$ Recently the orbifold compactification with magnetic fluxes was studied within the framework of heterotic string theory [20].

${ }^{3}$ Here, we consider the twist orbifolding, although shift orbifolding is also possible [21].
} 


\section{The 10D SYM theory in $\mathcal{N}=1$ superspace}

We consider a compactification of 10D SYM theory on 4D flat Minkowski spacetime times a product of factorizable three tori $T^{2} \times T^{2} \times T^{2}$ using the superfield description suitable for such a compactification with magnetic fluxes preserving $4 \mathrm{D} \mathcal{N}=1$ SUSY. Basically, we assume that the compactification scale is around the GUT or Planck scale, although application to other compactifications is straightforward. We mostly follow the notations and the conventions adopted in our previous works $[14,15]$. Here, we briefly review only essential points for this work.

We decompose the 10D vector (gauge) field $A_{M}$ into the $4 \mathrm{D}$ vector field $A_{\mu}$ and three complex fields $A_{i}(i=1,2,3)$. Also the 10D Majorana-Weyl spinor field $\lambda$ is decomposed into four $4 \mathrm{D}$ Weyl spinor fields $\lambda_{0}$ and $\lambda_{i}(i=1,2,3)$. For $4 \mathrm{D}$ positive chirality, these spinor fields $\lambda_{0}, \lambda_{1}, \lambda_{2}$, and $\lambda_{3}$ have the $6 \mathrm{D}$ chiralities, $(+,+,+),(+,-,-),(-,+,-)$, and $(-,-,+)$ on $T^{2} \times T^{2} \times T^{2}$. The 10D SYM theory possesses $\mathcal{N}=4$ SUSY in terms of $4 \mathrm{D}$ supercharges. According to that, the YM vector and spinor fields of 10D SYM theory are decomposed into (on-shell) $4 \mathrm{D} \mathcal{N}=1$ single vector and triple chiral multiplets, $\boldsymbol{V}=\left\{A_{\mu}, \lambda_{0}\right\}$ and $\phi_{i}=\left\{A_{i}, \lambda_{i}\right\}(i=1,2,3)$. We assign them to vector and chiral superfields, $V$ and $\phi_{i}$, respectively. Using them, the 10D SYM action can be written in the $\mathcal{N}=1$ superspace as $[14,22,23]$

$$
S=\int d^{10} X \sqrt{-G}\left[\int d^{4} \theta \mathcal{K}+\left\{\int d^{2} \theta\left(\frac{1}{4 g^{2}} \mathcal{W}^{\alpha} \mathcal{W}_{\alpha}+\mathcal{W}\right)+\text { h.c. }\right\}\right] .
$$

$\mathcal{K}, \mathcal{W}$ and $\mathcal{W}_{\alpha}$ are functions of the superfields

$$
\begin{aligned}
\mathcal{K} & =\frac{2}{g^{2}} h^{\bar{i} j} \operatorname{Tr}\left[\left(\sqrt{2} \bar{\partial}_{i}+\bar{\phi}_{\bar{i}}\right) e^{-V}\left(-\sqrt{2} \partial_{j}+\phi_{j}\right) e^{V}+\bar{\partial}_{\bar{i}} e^{-V} \partial_{j} e^{V}\right]+\mathcal{K}_{\mathrm{WZW}} \\
\mathcal{W} & =\frac{1}{g^{2}} \epsilon^{\mathrm{ijk}} e_{\mathrm{i}}{ }^{i} e_{\mathrm{j}}{ }^{j} e_{\mathrm{k}}{ }^{k} \operatorname{Tr}\left[\sqrt{2} \phi_{i}\left(\partial_{j} \phi_{k}-\frac{1}{3 \sqrt{2}}\left[\phi_{j}, \phi_{k}\right]\right)\right] \\
\mathcal{W}_{\alpha} & =-\frac{1}{4} \bar{D} \bar{D} e^{-V} D_{\alpha} e^{V}
\end{aligned}
$$

where $\partial_{i}$ denotes a derivative of complex coordinate $z_{i}$, and $h^{\bar{i} j}$ and $e_{\mathrm{i}}{ }^{i}$ are metric and vielbein of the $i$-th torus. The term $\mathcal{K}_{\text {WZW }}$ denotes a Wess-Zumino-Witten term which vanishes in the Wess-Zumino (WZ) gauge. Note that only the combination $\phi_{1} \phi_{2} \phi_{3}$ has nonvanishing Yukawa couplings in $\mathcal{W}$.

The field equations for auxiliary fields $D$ and $F_{i}$ are

$$
\begin{aligned}
& D=-h^{\bar{i} j}\left(\bar{\partial}_{\bar{i}} A_{j}+\partial_{j} \bar{A}_{\bar{i}}+\frac{1}{2}\left[\bar{A}_{\bar{i}}, A_{j}\right]\right), \\
& \bar{F}_{\bar{i}}=-h_{j \bar{i}} \epsilon^{\mathrm{jkl}} e_{\mathrm{j}}^{j} e_{\mathrm{k}}^{k} e_{\mathrm{l}}^{l}\left(\partial_{k} A_{l}-\frac{1}{4}\left[A_{k}, A_{l}\right]\right) .
\end{aligned}
$$

The condition $\langle D\rangle=\left\langle F_{i}\right\rangle=0$ determines SUSY vacua. A trivial one is given by $\left\langle A_{i}\right\rangle=0$, where the full $\mathcal{N}=4$ SUSY as well as the YM gauge symmetry is preserved. In the following, we search nontrivial SUSY vacua where magnetic fluxes exist in the YM sector. 


\section{Magnetic fluxes and zero-modes}

We consider the 10D U $(N)$ SYM theory ${ }^{4}$ on a SUSY background with factorizable magnetic fluxes. In this section, we study the case that the YM fields take the following vacuum expectation values (VEVs), which have $4 \mathrm{D}$ Lorentz invariant and at least $\mathcal{N}=1$ SUSY (with the conditions (3.3) and (3.4)),

$$
\left\langle A_{i}\right\rangle=\frac{\pi}{\operatorname{Im} \tau_{i}}\left(M^{(i)} \bar{z}_{\bar{i}}+\bar{\zeta}_{i}\right), \quad\left\langle A_{\mu}\right\rangle=\left\langle\lambda_{0}\right\rangle=\left\langle\lambda_{i}\right\rangle=\left\langle F_{i}\right\rangle=\langle D\rangle=0 .
$$

This VEV of $A_{i}$ leads to factorizable magnetic fluxes, and this form is unique up to gauge transformation for the fixed magnitude of $M^{(i)}$. Here $M^{(i)}$ denotes the $N \times N$ diagonal matrices of Abelian magnetic fluxes as

$$
M^{(i)}=\left(\begin{array}{llll}
M_{1}^{(i)} \mathbf{1}_{N_{1}} & & & \\
& M_{2}^{(i)} \mathbf{1}_{N_{2}} & & \\
& & & \ddots \\
& & & M_{n}^{(i)} \mathbf{1}_{N_{n}}
\end{array}\right)
$$

where $\mathbf{1}_{N_{a}}$ is a $N_{a} \times N_{a}$ unit matrix. Similarly, the Wilson-lines $\zeta_{i}$ are denoted as

$$
\zeta^{(i)}=\left(\begin{array}{llll}
\zeta_{1}^{(i)} \mathbf{1}_{N_{1}} & & & \\
& \zeta_{2}^{(i)} \mathbf{1}_{N_{2}} & & \\
& & \ddots & \\
& & & \zeta_{n}^{(i)} \mathbf{1}_{N_{n}}
\end{array}\right)
$$

$\tau_{i}$ is complex structure of the $i$-th torus. We restrict $\tau_{i}$ to be pure imaginary, since its real part only affects physical CP phases. The magnetic fluxes satisfy the Dirac's quantization condition, $M_{1}^{(i)}, M_{2}^{(i)}, \ldots, M_{n}^{(i)} \in \boldsymbol{Z}$. Besides, they are constrained by the SUSY conditions $\langle D\rangle=0$ and $\left\langle F_{i}\right\rangle=0$ in eq. (3.1) which lead

$$
\begin{aligned}
h^{\bar{i} j}\left(\bar{\partial}_{\bar{i}}\left\langle A_{j}\right\rangle+\partial_{j}\left\langle\bar{A}_{\bar{i}}\right\rangle\right) & =0, \\
\epsilon^{\mathrm{jkl}} e_{\mathrm{k}}^{k} e_{\mathrm{l}}^{l} \partial_{k}\left\langle A_{l}\right\rangle & =0,
\end{aligned}
$$

with $D$ and $F_{i}$ given by eqs. (2.1) and (2.2), respectively. Magnetic fluxes with the VEVs eq. (3.1) trivially satisfy the $\mathrm{F}$ term condition eq. (3.4).

The magnetic flux (3.2) breaks the $\mathrm{U}(N)$ gauge symmetry into $\prod_{a} \mathrm{U}\left(N_{a}\right)$ for $a=$ $1,2, \ldots, n$ if all the magnetic fluxes $M_{1}^{(i)}, M_{2}^{(i)}, \ldots, M_{n}^{(i)}$ take values different from each other. The same holds for Wilson-lines, $\zeta_{1}^{(i)}, \zeta_{2}^{(i)}, \ldots, \zeta_{n}^{(i)}$. In the following, the indices $a, b=1,2, \ldots, n$ label the YM subgroups unbroken by the fluxes and Wilson-lines, and traces in expressions are performed within such subgroups.

On the $\mathcal{N}=1$ SUSY toroidal background (3.1) with the magnetic fluxes and the Wilson-lines, the zero-modes $\left(V^{\boldsymbol{n}=\mathbf{0}}\right)_{a b}$ of the off-diagonal elements $(V)_{a b}(a \neq b)$ become

\footnotetext{
${ }^{4} \mathrm{~A}$ similar study with other gauge groups is possible [24, 25].
} 
massive, while the diagonal elements $\left(V^{\boldsymbol{n}=\mathbf{0}}\right)_{a a}$ do not. Then, we denote the zero-modes $\left(V^{\boldsymbol{n}=\mathbf{0}}\right)_{a a}$, which contain $4 \mathrm{D}$ gauge fields for the unbroken gauge symmetry $\mathrm{U}\left(N_{a}\right)$, as

$$
\left(V^{\boldsymbol{n}=\mathbf{0}}\right)_{a a} \equiv V^{a}
$$

The magnetic fluxes and the Wilson-lines have no effect on these diagonal elements, and extra dimensional parts of their wavefunctions are constant. On the other hand, for ${ }^{\exists} j \neq i$ with $M_{a b}^{(j)} \equiv M_{a}^{(j)}-M_{b}^{(j)}>0, M_{a b}^{(i)} \equiv M_{a}^{(i)}-M_{b}^{(i)}<0$ and $a \neq b$, the zero-mode $\left(\phi_{j}^{\mathbf{n}=\mathbf{0}}\right)_{a b}$ of the off-diagonal element $\left(\phi_{j}\right)_{a b}$ degenerates with the number of degeneracy

$$
N_{a b}=\prod_{k}\left|M_{a b}^{(k)}\right|
$$

Then, $\left(\phi_{j}^{\boldsymbol{n}=\mathbf{0}}\right)_{b a}$ has no zero-mode solution, that is, the magnetic fluxes yield a $4 \mathrm{D}$ SUSY chiral generation in the $a b$-sector [14]. Since there is one zero-mode without fluxes on a torus, eq. (3.5) is not valid if any one of $M_{a b}^{(k)}$ is zero. Practically, such a situation appears later and we will elaborate on that there.

We denote the zero-mode $\left(\phi_{j}^{\boldsymbol{n}=\mathbf{0}}\right)_{a b}$ with the degeneracy $N_{a b}$ as

$$
\left(\phi_{j}^{\boldsymbol{n}=\mathbf{0}}\right)_{a b} \equiv g \phi_{j}^{\mathcal{I}_{a b}, M_{a b}^{(i)}}
$$

where $\mathcal{I}_{a b}$ labels the degeneracy, i.e. generations, and we normalize it by the $10 \mathrm{D}$ YM coupling constant $g$. An extra dimensional part of their wavefunctions is decomposed into three parts and each of them corresponds to the $i$-th torus. These wavefunctions can be written by the Jacobi theta-function. For example, for the $i$-th torus, the zero-mode wavefunctions are written by

$$
\phi_{j}^{\mathcal{I}_{a b}^{(i)}, M_{a b}^{(i)}}\left(\tau_{i}, z_{i}\right)=\mathcal{N} \cdot e^{i \pi M_{a b}^{(i)} z_{i} \operatorname{Im} z_{i} / \operatorname{Im} \tau_{i}} \cdot \vartheta\left[\begin{array}{c}
\mathcal{I}_{a b}^{(i)} / M_{a b}^{(i)} \\
0
\end{array}\right]\left(M_{a b}^{(i)} z_{i}, M_{a b}^{(i)} \tau_{i}\right),
$$

where $\mathcal{I}_{a b}^{(i)}$ labels the degeneracy generated on the $i$-th torus and $\vartheta$ is the Jacobi thetafunction,

$$
\vartheta\left[\begin{array}{l}
a \\
b
\end{array}\right](\nu, \tau)=\sum_{l \in \mathbb{Z}} e^{\pi i(a+l)^{2} \tau} e^{2 \pi i(a+l)(\nu+b)} .
$$

The normalization factor $\mathcal{N}$ is defined as follows

$$
\int_{T^{2}} d z d \bar{z} \phi_{j}^{I, M}\left(\phi_{j}^{K, M}\right)^{*}=\delta_{I K}
$$

For more details, see ref. [14] and references therein. 


\subsection{Factorizable flux Ansatz}

We are going to get zero-mode spectra that contain the MSSM with the gauge group $\mathrm{SU}(3)_{C} \times \mathrm{SU}(2)_{L} \times \mathrm{U}(1)_{Y}$ and three generations of the quark and the lepton chiral multiplets, by identifying these three generations with three degenerate zero-modes of the chiral superfields $\phi_{j}^{\mathcal{I}_{a b}}$.

To obtain the SM gauge group, especially $\mathrm{U}(1)_{Y}$, we have to start at least the $10 \mathrm{D}$ $\mathrm{U}(8)$ SYM theory which contains the Pati-Salam gauge group, $\mathrm{U}(4)_{C} \times \mathrm{U}(2)_{L} \times \mathrm{U}(2)_{R}$, up to $\mathrm{U}(1)$ factors. In the Pati-Salam gauge group, $(4,2,1)$ matter fields correspond to lefthanded quarks and leptons, while $(\overline{4}, 1,2)$ matter fields correspond to right-handed quarks and leptons. In addition, Higgs fields are assigned to $(1,2,2)$ representations. Although we can start with a larger gauge group, we concentrate ourselves on the $\mathrm{U}(8)$ gauge group as our starting point.

In our models, magnetic fluxes yield potentially hierarchical Yukawa couplings from $10 \mathrm{D}$ gauge couplings, the tri-linear terms in $\mathcal{W}$. We require full-rank Yukawa matrices among the three generations. If the flavor structure of left-handed and right-handed matter fields are originated from different tori, one obtains rank-one Yukawa matrices at the treelevel. It leads two massless generations (at the tree-level). That cannot be realistic unless there are corrections such as nonperturbative effects. We will derive the three generation structures of all the chiral matter fields on the first torus to obtain full-rank Yukawa matrices.

Now, we study proper configurations of Abelian factorizable magnetic fluxes. To obtain three generation structures on the first torus, we introduce fluxes with $M_{a b}^{(1)}=3$ for the quark and lepton chiral multiplets. The simplest one is

$$
M^{(1)}=\left(\begin{array}{ccc}
0 \times \mathbf{1}_{4} & \\
& 3 \times \mathbf{1}_{4}
\end{array}\right) .
$$

Recall that $(4,2,1)$ and $(\overline{4}, 1,2)$ correspond to quarks and leptons. Since only differences between magnetic fluxes have effects on physics, we fix the first element along the U(1) direction of $\mathrm{U}(4)_{C}$ to be vanishing. We set $M^{(2)}$ and $M^{(3)}$ such that they do not generate extra generations and satisfy SUSY preserving conditions eqs. (3.3) and (3.4). In addition, the gauge group is to be further broken down to $\mathrm{U}(3)_{C} \times \mathrm{U}(1)_{C^{\prime}} \times \mathrm{U}(2)_{L} \times \mathrm{U}(1)_{R^{\prime}} \times \mathrm{U}(1)_{R^{\prime \prime}}$ by the following Wilson-lines,

$$
\zeta_{1}=\left(\begin{array}{ccccc}
\zeta_{C}^{(1)} \mathbf{1}_{3} & & & & \\
& \zeta_{C^{\prime}}^{(1)} & & & \\
& & \zeta_{L}^{(1)} \mathbf{1}_{2} & & \\
& & & \zeta_{R^{\prime}}^{(1)} & \\
& & & & \zeta_{R^{\prime \prime}}^{(1)}
\end{array}\right)
$$

where all the nonvanishing entries take values different from each other. However, in this two-block Ansatz (3.8), the Higgs fields with the $(1,2,2)$ representation have no effects due to magnetic fluxes, and their zero-mode profiles are just flat. Thus, the Yukawa matrices 
are proportional to the $(3 \times 3)$ unit matrix and that is not realistic. ${ }^{5}$ To construct the plausible models which have three generation matter fields and full-rank Yukawa matrices, more complicated fluxes are needed.

The next-to-simplest one is as follows,

$$
M^{(1)}=\left(\begin{array}{cccc}
0 \times \mathbf{1}_{4} & & \\
& 3 \times \mathbf{1}_{2} & \\
& & & -3 \times \mathbf{1}_{2}
\end{array}\right) .
$$

We also set $M^{(2)}$ and $M^{(3)}$ such that they do not generate extra generations and satisfy SUSY preserving conditions eqs. (3.3) and (3.4). The gauge group is broken as $\mathrm{U}(8) \rightarrow$ $\mathrm{U}(4)_{C} \times \mathrm{U}(2)_{L} \times \mathrm{U}(2)_{R}$, the Pati-Salam gauge group, which is further broken by the Wilsonlines eq. (3.9) in a similar way. This type of magnetic fluxes, the three-block type, will be studied in detail in the following sections.

We can consider more complicated flux configurations such as the four-block or fiveblock magnetic fluxes, where $\mathrm{U}(4)_{C}$ is broken as $\mathrm{U}(4)_{C} \rightarrow \mathrm{U}(3)_{C} \times \mathrm{U}(1)_{C}^{\prime}$ and/or $\mathrm{U}(2)_{R}$ is broken as $\mathrm{U}(2)_{R} \rightarrow \mathrm{U}(1)_{R^{\prime}} \times \mathrm{U}(1)_{R^{\prime \prime}}$ by magnetic fluxes. However, in those models one can not obtain full-rank Yukawa matrices satisfying the SUSY conditions. Thus, we do not consider such possibilities, and we concentrate on the three-block Ansatz of magnetic fluxes (3.10) with the Wilson-lines eq. (3.9).

\subsection{Pati-Salam model}

We show the details of the Pati-Salam models in which the flavor structures are originated by the flux eq. (3.10). The Pati-Salam gauge group generated by the flux is further broken down to $\mathrm{U}(3)_{C} \times \mathrm{U}(1)_{C^{\prime}} \times \mathrm{U}(2)_{L} \times \mathrm{U}(1)_{R^{\prime}} \times \mathrm{U}(1)_{R^{\prime \prime}}$ by the Wilson-lines (3.9). The gauge symmetries $\mathrm{SU}(3)_{C}$ and $\mathrm{SU}(2)_{L}$ of the MSSM can be embedded into the unbroken gauge subgroups, as $\mathrm{SU}(3)_{C} \subset \mathrm{U}(3)_{C}$ and $\mathrm{SU}(2)_{L} \subset \mathrm{U}(2)_{L}$. The hypercharge $\mathrm{U}(1)_{Y}$ is obtained as a proper linear combinations of $\mathrm{U}(1)$ 's.

We show all the possible patterns of magnetic flux configuration satisfying SUSY preserving condition eqs. (3.3) and (3.4) as a result of systematic search in table 1 by using the following notation:

$$
M^{(i)}=\left(\begin{array}{cccc}
M_{C}^{(i)} \mathbf{1}_{4} & & & \\
& M_{L}^{(i)} \mathbf{1}_{2} & \\
& & & \\
& & M_{R}^{(i)} \mathbf{1}_{2}
\end{array}\right) .
$$

There are three patterns and one of them, pattern 1, is studied in ref. [15]. These three lead phenomenological features quite similar to each other, thus, we show the details of the first one in the following.

The first one flux configuration satisfies the SUSY preserving conditions eqs. (3.3) and (3.4) with

$$
\mathcal{A}^{(1)} / \mathcal{A}^{(2)}=\mathcal{A}^{(1)} / \mathcal{A}^{(3)}=3,
$$

\footnotetext{
${ }^{5}$ By introducing Wilson-lines, the zero-mode profiles of the Higgs fields become nontrivial and Yukawa couplings may be modified [26]. However, such Wilson-lines make the Higgs fields massive, and the Wilsonlines corresponding to $\mathcal{O}(100) \mathrm{GeV}$ mass scale would not lead sufficient modification to lead realistic results.
} 


\begin{tabular}{|c|c|c|c|}
\hline & $\left(M_{C}^{(1)}, M_{L}^{(1)}, M_{R}^{(1)}\right)$ & $\left(M_{C}^{(2)}, M_{L}^{(2)}, M_{R}^{(2)}\right)$ & $\left(M_{C}^{(3)}, M_{L}^{(3)}, M_{R}^{(3)}\right)$ \\
\hline pattern 1 & $(0,+3,-3)$ & $(0,-1,0)$ & $(0,0,+1)$ \\
\hline pattern 2 & $(0,+3,-3)$ & $(0,-1,0)$ & $(0,+1,+1)$ \\
\hline pattern 3 & $(0,+3,-3)$ & $(0,-1,-1)$ & $(0,0,+1)$ \\
\hline
\end{tabular}

Table 1. Three patterns to give the Pati-Salam model

where $\mathcal{A}^{(i)}$ is an area of the $i$-th torus. In this case, all the matter supermultiplets and Higgs supermultiplets of the MSSM, $Q, U, D, L, N, E, H_{u}$ and $H_{d}$, are found in $\phi_{i}^{\mathcal{I}_{a b}}$ as

$$
\begin{aligned}
& \phi_{1}^{\mathcal{I}_{a b}}=\left(\begin{array}{cc|c|cc}
\Omega_{C}^{(1)} & \Xi_{C C^{\prime}}^{(1)} & 0 & \Xi_{C R^{\prime}}^{(1) J} & \Xi_{C R^{\prime \prime}}^{(1) J} \\
\Xi_{C^{\prime} C}^{(1)} & \Omega_{C^{\prime}}^{(1)} & 0 & \Xi_{C^{\prime} R^{\prime}}^{(1) J} & \Xi_{C^{\prime} R^{\prime \prime}}^{(1) J} \\
\hline \Xi_{L C}^{(1) I} & \Xi_{L C^{\prime}}^{(1) I} & \Omega_{L}^{(1)} & H_{u}^{K} & H_{d}^{K} \\
\hline 0 & 0 & 0 & \Omega_{R^{\prime}}^{(1)} & \Xi_{R^{\prime} R^{\prime \prime}}^{(1)} \\
0 & 0 & 0 & \Xi_{R^{\prime \prime} R^{\prime}}^{(1)} & \Omega_{R^{\prime \prime}}^{(1)}
\end{array}\right), \\
& \phi_{2}^{\mathcal{I}_{a b}}=\left(\begin{array}{cc|c|cc}
\Omega_{C}^{(2)} & \Xi_{C C^{\prime}}^{(2)} & Q^{I} & 0 & 0 \\
\Xi_{C^{\prime} C}^{(2)} & \Omega_{C^{\prime}}^{(2)} & L^{I} & 0 & 0 \\
\hline 0 & 0 & \Omega_{L}^{(2)} & 0 & 0 \\
\hline 0 & 0 & 0 & \Omega_{R^{\prime}}^{(2)} & \Xi_{R^{\prime} R^{\prime \prime}}^{(2)} \\
0 & 0 & 0 & \Xi_{R^{\prime \prime} R^{\prime}}^{(2)} & \Omega_{R^{\prime \prime}}^{(2)}
\end{array}\right) \text {, } \\
& \phi_{3}^{\mathcal{I}_{a b}}=\left(\begin{array}{cc|c|cc}
\Omega_{C}^{(3)} & \Xi_{C C^{\prime}}^{(3)} & 0 & 0 & 0 \\
\Xi_{C^{\prime} C}^{(3)} & \Omega_{C^{\prime}}^{(3)} & 0 & 0 & 0 \\
\hline 0 & 0 & \Omega_{L}^{(3)} & 0 & 0 \\
\hline U^{J} & N^{J} & 0 & \Omega_{R^{\prime}}^{(3)} & \Xi_{R^{\prime} R^{\prime \prime}}^{(3)} \\
D^{J} & E^{J} & 0 & \Xi_{R^{\prime \prime} R^{\prime}}^{(3)} & \Omega_{R^{\prime \prime}}^{(3)}
\end{array}\right) \text {, }
\end{aligned}
$$

where both of the rows and the columns of matrices correspond to $a, b=C, C^{\prime}, L, R^{\prime}, R^{\prime \prime}$, and the indices $I, J=1,2,3$ and $K=1, \ldots, 6$ label the degenerate zero-modes, i.e., generations. As a result, three anti-generation fields are generated in some conjugate sectors of the MSSM contents as well as the MSSM contents. For example, $\Xi_{L C}^{(1)}$ and $\Xi_{L C^{\prime}}^{(1)}$ are anti-generation fields of the left-handed matter fields. Anti-generations have charges and feel magnetic fluxes, both of which are opposite to their corresponding MSSM contents. Note that, since anti-generations feel magnetic fluxes whose absolute values are the same as those of the corresponding MSSM fields, their degeneracy are the same.

Now we have vector-like matter fields. To explain that, we show the details of magnetic fluxes which each representation feels on each torus in table 2.

First, the Higgs sector feels the fluxes $(+,-,-)$ on the three tori. After the chirality projection caused by magnetic fluxes, they survive in only $\phi_{1}$ which have the $6 \mathrm{D}$ chirality $(+,-,-)$ and their degeneracy is given by eq. (3.5), i.e., six generations. Note that the fields, which have charges opposite to $H_{u}^{K}$ and $H_{d}^{K}$, would feel the magnetic fluxes, $(-,+,+)$ 


\begin{tabular}{|c|c|c|c|}
\hline & Left-handed & Higgs & Right-handed \\
\hline & $M_{C}^{(i)}-M_{L}^{(i)}$ & $M_{L}^{(i)}-M_{R}^{(i)}$ & $M_{R}^{(i)}-M_{C}^{(i)}$ \\
\hline 1st $T^{2}$ & -3 & 6 & -3 \\
2nd $T^{2}$ & +1 & -1 & 0 \\
3rd $T^{2}$ & 0 & -1 & +1 \\
\hline
\end{tabular}

Table 2. Magnetic fluxes for each representation on each torus.

but they do not appear in the massless spectrum. ${ }^{6}$ On the other hand, the left-handed and right-handed matter sectors have a torus with vanishing flux. In this case, there is one zero-mode which has a flat wavefunction on such a torus and the total degeneracy is not given by eq. (3.5). Since the chirality projection does not work, their anti-generations also survive. That directly leads vector-like fields. To explain more explicitly, the left-handed sector feels the fluxes $(-,+, 0)$ on the three tori. They survive in $\phi_{2}$ which have the $6 \mathrm{D}$ chirality $(-,+,-)$ and their degeneracy is given by " $3 \times 1 \times 1$ ". Note that there is one zero-mode without fluxes on a torus. Then, their anti-generations feel the fluxes $(+,-, 0)$ and survive in $\phi_{1}$ with the same degeneracy " $3 \times 1 \times 1$ ". The right-handed sector becomes vector-like similarly.

In order to realize three generations of quarks and leptons with the full-rank Yukawa coupling matrices, some of $M_{a b}^{(i)}$ would be zero for any of three patterns shown in table 1. It is inevitable that two of the three sectors become vector-like. Furthermore, all the three patterns lead massless diagonal components $\Omega_{a}^{(r)}$, the so-called open string moduli, which also feel zero fluxes.

However, most of these extra contents, anti-generations and open string moduli, can be projected out with the three generations of quarks and leptons unchanged if we impose further a certain orbifold projection on the second and third tori [15]. ${ }^{7}$ After such a certain orbifold projection, the three patterns of flux configurations give exactly the same zero-mode contents including some of the exotic modes and open string moduli.

The orbifold projection is very useful to remove the extra modes. Furthermore, considering orbifold projections on the first and second tori (or the first and third tori), there are some possibilities to obtain three generation structure with magnetic fluxes other than we showed above and we study such possibilities in the next section.

One can generalize the above results for $\mathrm{N}$ generations of the chiral matter fields by replacing " $3 \rightarrow \mathrm{N}$ ". We strongly owe this generalization to the constraint that we have to derive generation structures on only the first torus to obtain full-rank Yukawa matrices.

\section{$3.3 \quad Z_{2}$ projection}

We consider the $Z_{2}$ projection, and then either even or odd modes of zero-modes remain. Zero-mode wavefunctions have the following relation [19],

$$
\phi^{I}(-z)=\phi^{M-I}(z) .
$$

\footnotetext{
${ }^{6}$ The 10D Majorana-Weyl spinor field does not include the 4D Weyl spinor field which have the corresponding $6 \mathrm{D}$ chirality, $(-,+,+)$.

${ }^{7}$ We show that in appendix A.
} 


\begin{tabular}{|cccccccccccc|}
\hline$M$ & 0 & 1 & 2 & 3 & 4 & 5 & 6 & 7 & 8 & 9 & 10 \\
\hline even & 1 & 1 & 2 & 2 & 3 & 3 & 4 & 4 & 5 & 5 & 6 \\
odd & 0 & 0 & 0 & 1 & 1 & 2 & 2 & 3 & 3 & 4 & 4 \\
\hline
\end{tabular}

Table 3. The numbers of zero-modes for even and odd wavefunctions.

Using that, even and odd functions are given by,

$$
\begin{aligned}
& \phi_{\text {even }}^{I}(z)=\frac{1}{\sqrt{2}}\left(\phi^{I}(z)+\phi^{M-I}(z)\right), \\
& \phi_{\text {odd }}^{I}(z)=\frac{1}{\sqrt{2}}\left(\phi^{I}(z)-\phi^{M-I}(z)\right) .
\end{aligned}
$$

The degeneracy of zero-modes after the $Z_{2}$ projection is changed, and we show that in table 3 [19], where $M=M_{a b}^{(r)}$ on each $r$-th torus.

One way to construct the plausible models by using the $T^{6} / Z_{2}$ orbifold is that we start with magnetic fluxes shown in table 1 and then we assume the $T^{6} / Z_{2}$ orbifold where the $Z_{2}$ acts on the second and the third tori $r=2,3$. In this model building, we can eliminate extra modes without affecting three generations of quarks and leptons, as shown in appendix A and ref. [15].

On the other hand, from table 3 , we can see that there are other possibilities to obtain three generation structures by assuming that the $Z_{2}$ acts on the first and the second tori $r=1,2$, or the first and third tori $r=1,3: M=4,5$ for even modes and $M=7,8$ for odd modes. Here we study such possibilities. We concentrate on the case that the $Z_{2}$ projection acts on the first and the second tori $r=1,2$ assuming that three generations are originated from the first torus, $r=1$. That is, the degeneracy numbers of quark and lepton zero-modes are equal to three for $r=1$ after $Z_{2}$ orbifolding, while the degeneracy numbers are equal to one for $r=2$ after orbifolding and one for the third torus. Then, $Z_{2}$ boundary conditions of 10D superfields $V$ and $\phi_{i}$ are assigned as

$$
\begin{aligned}
V\left(x,-y_{m}, y_{n}\right) & =+P V\left(x, y_{m}, y_{n}\right) P^{-1}, \\
\phi_{1}\left(x,-y_{m}, y_{n}\right) & =-P \phi_{1}\left(x, y_{m}, y_{n}\right) P^{-1}, \\
\phi_{2}\left(x,-y_{m}, y_{n}\right) & =-P \phi_{2}\left(x, y_{m}, y_{n}\right) P^{-1}, \\
\phi_{3}\left(x,-y_{m}, y_{n}\right) & =+P \phi_{3}\left(x, y_{m}, y_{n}\right) P^{-1},
\end{aligned}
$$

for ${ }^{\forall} m=4,5,6,7$ and ${ }^{\forall} n=8,9$, where $P$ is a projection operator acting on YM indices satisfying $P^{2}=\mathbf{1}_{N}$. The $\phi_{1}$ and $\phi_{2}$ have the minus sign under the $Z_{2}$ reflection, because they are the vector fields $A_{i}(i=1,2)$ on the $Z_{2}$ orbifold plane (the first and the second tori). Note that the orbifold projection (3.13) respects the $\mathcal{N}=1$ SUSY preserved by the magnetic fluxes table 1 , because the $Z_{2}$-parities are assigned to each of the $\mathcal{N}=1$ superfields $V$ and $\phi_{i}$.

Choosing a proper projection operator $P$, we can construct the Pati-Salam models eq. (3.10) with three generation chiral matter fields other than we showed in table 1. However, on account of the orbifold projection (3.13), nonvanishing (continuous) Wilson- 
line parameters eq. (3.9) are possible only on the third torus. ${ }^{8}$ The Pati-Salam gauge group can be broken down as shown previously by the Wilson-lines eq. (3.9) on the third torus. Recall that the three generation structures are originated on the first torus. Therefore, with the above type of three-block magnetic fluxes, all the Yukawa matrices for the up-sector and down-sector of quarks, neutrinos, and charged leptons, have exactly the same form except a universal factor. The experimental values of their masses and mixings cannot be realized. Thus, we need to consider the following five-block magnetic fluxes

$$
M^{(i)}=\left(\begin{array}{ccccc}
M_{C}^{(i)} \mathbf{1}_{3} & & & & \\
& M_{C^{\prime}}^{(i)} & & & \\
& & M_{L}^{(i)} \mathbf{1}_{2} & & \\
& & & M_{R^{\prime}}^{(i)} & \\
& & & & M_{R^{\prime \prime}}^{(i)}
\end{array}\right),
$$

where all the nonvanishing entries take values different from each other on at least the first tori. We search such a possibility in a systematic way. Then, it is found that it is impossible to construct three generation models with orbifold projections and five-block fluxes (3.14), since the SUSY preserving conditions become severer than the case with three-block fluxes. Obviously, that is the same in the case that the $Z_{2}$ acts on the first and the third tori $r=1,3$.

So far, we can obtain a unique flavor structure, which was studied in ref. [15]. We have considered only the simple fluxes like eq. (3.1). However, nonfactorizable fluxes which are magnetic fluxes crossing over two tori are studied in ref. [5, 16, 17], and it may enable us to obtain more various flavor structures and we study that in the next section.

\section{Nonfactorizable fluxes}

In this section, we study nonfactorizable flux models, where magnetic fluxes cross over two tori. We consider the case that the YM fields $A_{i}$ take the following VEVs instead of eq. (3.1)

$$
\begin{aligned}
\left\langle A_{i}\right\rangle & =\frac{\pi}{\operatorname{Im} \tau_{i}} M^{(i)} \bar{z}_{\bar{i}}+\frac{\pi}{\operatorname{Im} \tau_{j}} M^{(i j)} \bar{z}_{\bar{j}}+\bar{\zeta}_{i}, \\
\left\langle A_{\mu}\right\rangle & =\left\langle\lambda_{0}\right\rangle=\left\langle\lambda_{i}\right\rangle=\left\langle F_{i}\right\rangle=\langle D\rangle=0,
\end{aligned}
$$

where $i \neq j$. This is a straightforward extension of eq. (3.1) and the additional second term of the VEV of $A_{i}$ corresponds to (Abelian) nonfactorizable fluxes,

$$
M^{(i j)}=\left(\begin{array}{cccc}
M_{1}^{(i j)} \mathbf{1}_{N_{1}} & & & \\
& M_{2}^{(i j)} \mathbf{1}_{N_{2}} & & \\
& & & \ddots \\
& & & M_{n}^{(i j)} \mathbf{1}_{N_{n}}
\end{array}\right)
$$

\footnotetext{
${ }^{8}$ It is also possible to consider Wilson-lines in the first and the second tori, if we allow it to be constants changing their sign across the fixed points (planes) of the orbifold, that is beyond the scope of this paper.
} 
The VEV eq. (4.1) trivially satisfies the F term condition eq. (3.4) as well as the VEV eq. (3.1) does. That is because the complex structure in the second term of the first line in eq. (4.1) is consistent with the one in the first term. If we take a different coefficient in the second term, the SUSY condition is not satisfied.

For magnetic fluxes crossing over the two tori, e.g. the first and second tori, there are four elements, $F_{x_{1}, x_{2}}, F_{x_{1}, y_{2}}, F_{y_{1}, x_{2}}$, and $F_{y_{1}, y_{2}}$ in the real basis, where $z_{1}=\frac{1}{2}\left(x_{1}+\tau_{1} y_{1}\right)$ for the first torus and $z_{2}=\frac{1}{2}\left(x_{2}+\tau_{2} y_{2}\right)$ for the second torus. In order to derive a well defined wavefunction, only $F_{x_{1}, y_{2}}$ and $F_{y_{1}, x_{2}}$ are allowed with a certain SUSY relation [5, 16]. The VEV eq. (4.1) corresponds to such a case exactly.

With the above nonfactorizable fluxes, the numbers of degenerate zero-modes $\left(V^{n=0}\right)_{a b}$ and $\left(\phi_{j}^{n=0}\right)_{a b}$ are changed. $\left(V^{n=0}\right)_{a b}$ obtain mass terms with gauge symmetry breaking, so we explain about only chiral superfields $\phi_{j}$. For the moment, we consider only the case $i, j=1,2$ and $M^{(12)}$ and $M^{(21)}$ cross over between the first and the second tori. Later we will discuss its extensions, but such extensions do not lead to interesting models.

Now, we define the matrix

$$
\begin{aligned}
\mathcal{M}_{a b} & =\left(\begin{array}{cc}
m_{a b}^{(1)} & m_{a b}^{(12)} \\
m_{a b}^{(21)} & m_{a b}^{(2)}
\end{array}\right), \\
m_{a b}^{(i)} & =M_{a}^{(i)}-M_{b}^{(i)} \\
m_{a b}^{(i j)} & =m_{a}^{(i j)}-m_{b}^{(i j)} \\
m_{a}^{(i j)} & =\frac{\operatorname{Im} \tau_{i}}{\operatorname{Im} \tau_{j}} M_{a}^{(i j)}+M_{a}^{(j i)}
\end{aligned}
$$

Diagonal elements $m_{a b}^{(i)}$ correspond to the fluxes introduced in section 3, and nonvanishing off-diagonals to nonfactorizable fluxes. We have to impose Riemann conditions in order to have a well-defined zero-mode wavefunction, and then the matrix $\mathcal{M}$ and complex structure $\tau_{i}$ have to satisfy the following constraints [5]

$$
\mathcal{M}^{i j} \in \boldsymbol{Z}, \quad(\mathcal{M} \cdot \operatorname{Im} \Omega)^{T}=\mathcal{M} \cdot \operatorname{Im} \Omega, \quad \mathcal{M} \cdot \operatorname{Im} \Omega>0,
$$

where $\Omega \equiv \operatorname{diag}\left(\tau_{1}, \tau_{2}\right)$ is a general complex structure. ${ }^{9}$ Then, $\operatorname{det} \mathcal{M}$ determines the number of degenerate zero-modes on the two tori, and the total degeneracy is given by

$$
\left|\operatorname{det} \mathcal{M} \times M_{a b}^{(3)}\right| .
$$

This is valid as long as it is not zero, as well as eq. (3.5). While the zero-mode wavefunctions on the third torus are obtained by eq. (3.6) still, those on the other two tori are written by the following forms, ${ }^{10}$

$$
\phi^{\vec{j}_{a b}, \mathcal{M}_{a b}}(\vec{z}, \Omega)=\mathcal{N} \cdot e^{i \pi\left[\mathcal{M}_{a b} \cdot \vec{z}\right] \cdot\left(\mathcal{M}_{a b} \cdot \operatorname{Im} \Omega\right)^{-1} \operatorname{Im}\left[\mathcal{M}_{a b} \cdot \vec{z}\right]} \cdot \vartheta\left[\begin{array}{c}
\vec{j}_{a b} \\
0
\end{array}\right]\left(\mathcal{M}_{a b} \cdot \vec{z}, \mathcal{M}_{a b} \cdot \Omega\right),
$$

\footnotetext{
${ }^{9}$ In this paper, we unalterably focus on factorizable torus. We can consider generalized torus with off-diagonal elements of $\Omega[5,16,17]$, however, it is difficult to study that in a systematic way.

${ }^{10}$ Here, we take another gauge according to ref. [16]: $A_{i} \rightarrow A_{i}-\partial_{i} \chi^{(i)}$, where $\partial_{1} \chi^{(1)}=\frac{1}{\operatorname{Im} \tau_{2}} M^{(12)} \operatorname{Re} z_{2}$ and $\partial_{2} \chi^{(2)}=\frac{1}{\operatorname{Im} \tau_{1}} M^{(21)} \operatorname{Re} z_{1}$
} 
where $\vec{z}=\frac{1}{2}(\vec{x}+\Omega \vec{y}), \vec{j}_{a b}$ labels the degeneracy generated on the two tori, and $\vartheta$ is the Riemann theta-function, which is a generalization of the Jacobi theta-function eq. (3.7),

$$
\vartheta\left[\begin{array}{l}
\vec{a} \\
\vec{b}
\end{array}\right](\vec{\nu}, \Omega)=\sum_{\vec{m} \in \mathbb{Z}^{n}} e^{\pi(\vec{m}+\vec{a}) \cdot \Omega \cdot(\vec{m}+\vec{a})} e^{2 \pi i(\vec{m}+\vec{a}) \cdot(\vec{\nu}+\vec{b})} .
$$

Normalization is defined in a way similar to the previous one. For more details, see refs. [5, $16]$.

The constraints eq. (4.4) and the wavefunction eq. (4.6) are valid for a field $\phi_{3}$ which have (totally) positive chirality on the two tori. As for $\phi_{1}$ and $\phi_{2}$ which have the negative chirality $(+,-)$ or $(-,+)$ on the two tori, they need to be mixed up to get a zero-mode wavefunctions. Then, according to ref. [16], we parametrize them as follows,

$$
\phi_{1}^{a b}=\alpha^{a b} \Phi^{a b} \quad \phi_{2}^{a b}=\beta^{a b} \Phi^{a b} .
$$

The condition (4.4) to obtain a well-defined wavefunction and an explicit form of the wavefunction eq. (4.6) can also be applied to $\Phi$ with replacing $\Omega$ by $\tilde{\Omega}_{a b}=\hat{\Omega}_{a b} \cdot \Omega$, where

$$
\hat{\Omega}_{a b}=\frac{1}{1+q_{a b}^{2}}\left(\begin{array}{cc}
1-q_{a b}^{2} & -2 q_{a b} \\
-2 q_{a b} & q_{a b}^{2}-1
\end{array}\right) .
$$

Mixing parameters $q_{a b}=\beta_{a b} / \alpha_{a b}$ are given for individual bifundamental representations and their values are determined by the second one of eq. (4.4). Since $\operatorname{det} \hat{\Omega}=-1$, one can see $\operatorname{sign}(\operatorname{det} \mathcal{M})$ relates to the chirality of a field from the last one of eq. (4.4).

\subsection{Nonfactorizable flux Ansatz}

We study configurations of nonfactorizable magnetic fluxes. Similarly to the previous ones, we aim to realize a zero-mode spectrum in 10D magnetized U(8) SYM theory, that contains the MSSM again.

If there are only $M^{(i j)}$ fluxes with $M^{(i)}=0$, we obtain the same result as the factorizable models shown in section 3 with changing definition of torus cycles, so we consider both of nonvanishing $M^{(i)}$ and $M^{(i j)}$ simultaneously. In the previous section, we show the simplest case with nonfactorizable fluxes (4.1). Since we compactify the 10D SYM theory on three tori, one can think the magnetic flux background, where the VEVs of $A_{i}$ in eq. (4.2) have another term, $\frac{\pi}{\operatorname{Im} \tau_{k}} M^{(i k)} \bar{z}_{\bar{k}}$ for $k \neq i, j$. However, such general cases have strong constraints to realize a well-defined wavefunction, that is eq. (4.7). Indeed, although we search such general possibilities in a systematic way with all the flux parameters $M_{a}^{(i)}$ and $m_{a}^{(i j)}$ being in the range between -10 and 10 , we could not obtain three generation models in the parameter range. Thus, it is adequate for us to concentrate on the magnetic fluxes crossing over the first and second tori, eqs. (4.1) and (4.3). That is, we consider the cases, where nonvanishing magnetic fluxes correspond to $M^{(12)}, M^{(21)}$ and $M^{(i)}(i=1,2,3)$ while the others are vanishing. In the following, we search possible flux configurations in a systematic way, where the three generation structure is generated on the first and the second tori by the nonfactorizable fluxes. 
With the above setup, the three-block flux (Pati-Salam) model is the simplest. Twoblock models like eq. (3.2) are improbable in a way similar to the case with only the factorizable fluxes. In the three-block models, we need the Wilson-lines to break the PatiSalam gauge group as well as in section 3. If we could construct four-block or five-block models, e.g., like eq. (3.14), we would not necessarily need the Wilson-lines. We also search a possibility to give such an attractive model in the systematic way with all the flux parameters $M_{a}^{(i)}$ and $m_{a b}^{(i j)}$ being in the range between -10 and 10 , but we could not find a four-block model or a five-block model, because of the severer constraints for preserving SUSY and having well-defined wavefunctions, other than the Pati-Salam models . Thus, only the three-block Pati-Salam models are possible like the models with only the factorizable fluxes in section 3. In the next section, we show the details of our systematic research about the Pati-Salam models and its results.

\subsection{Pati-Salam model}

In this section, we concentrate on the three-block flux models with three (Abelian) factorizable fluxes $M^{(i)}(i=1,2,3)$ and (Abelian) nonfactorizable fluxes $M^{(12)}$ and $M^{(21)}$. We use $m_{a}^{(12)}$ and $m_{a}^{(21)}$ as parameters instead of $M_{a}^{(12)}$ and $M_{a}^{(21)}$, such as they satisfy the following relation

$$
\operatorname{Im} \tau_{1} m_{a}^{(21)}=\operatorname{Im} \tau_{2} m_{a}^{(12)}
$$

that is nothing but the SUSY preserving condition and satisfied automatically in eq. (4.3). There are an infinite number of possibilities, so we restrict the values of the parameters to the range between -10 and 10 . As a result, we find many possibilities for three generation models.

There are two types of models. The one, which we call "type 1", is that Higgs multiplets come from $\phi_{3}$ which have the chirality $(-,-,+)$. In this case left-handed and right-handed matter fields come from $\phi_{1}$ and $\phi_{2}$ both of which have the negative chirality totally on the first two tori. In the other case, "type2", either left-handed or right-handed matter fields come from $\phi_{3}$, and then they have the opposite chirality totally on the two tori.

First we show the result about the type1 models in figure 1. In this case, $M^{(3)}$ have the three possibilities, $M^{(3)}=\operatorname{diag}(0,1,-1), \operatorname{diag}(0,1,0)$ and $\operatorname{diag}(0,0,-1)$ without affecting the three generation structures of quark and lepton multiplets generated on the first and the second tori. The upper panel of figure 1 corresponds to the case with $M^{(3)}=\operatorname{diag}(0,1,-1)$. The number of Higgs pairs is increased twofold by $M^{(3)}$ on the third torus, and all the numbers of Higgs pairs are even. The lower panel shows the case that $M^{(3)}=\operatorname{diag}(0,1,0)$ and $\operatorname{diag}(0,0,-1)$.

We see that only particular numbers of Higgs pairs are allowed from the two panels. In the previous section, our three generation models inevitably include six pairs of Higgs multiplets. Now from the lower panel, we find the possibilities to obtain the minimal models with one pair of Higgs doublets just like the MSSM. It is quite attractive and we show the details in the next section. 

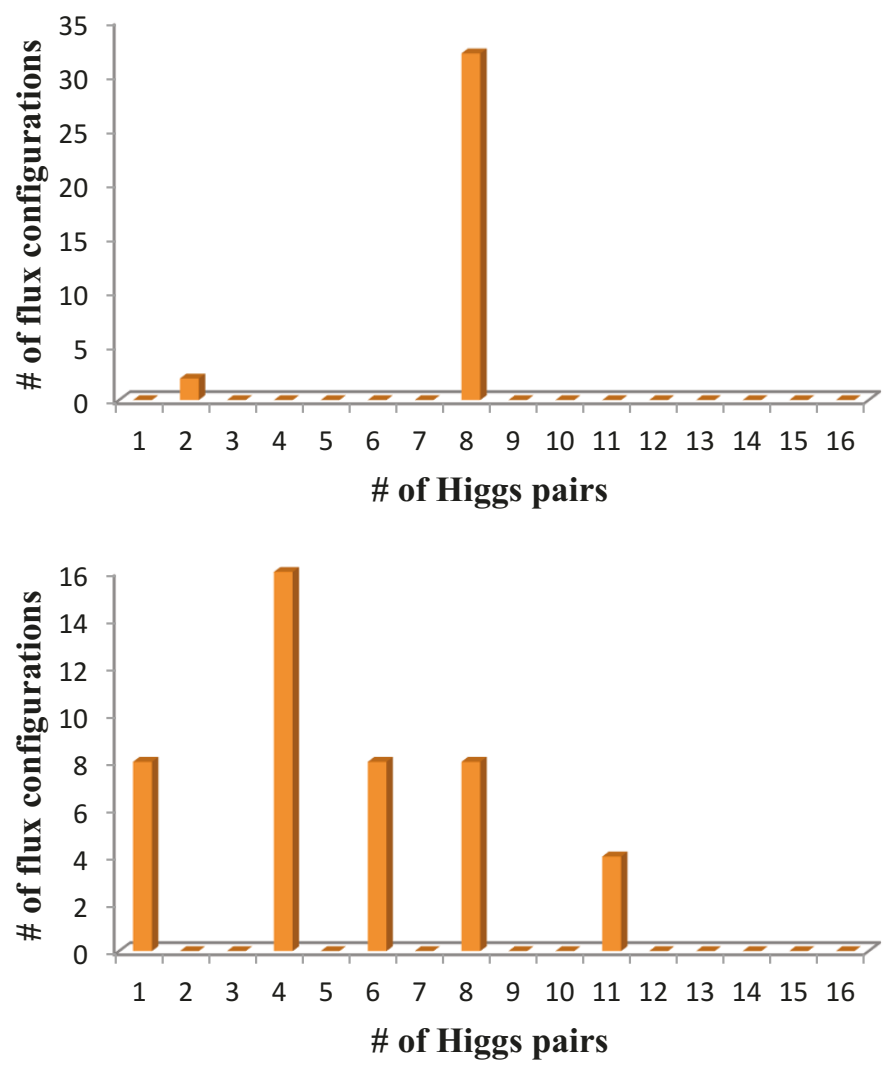

Figure 1. The number of magnetic flux configurations for each number of Higgs pairs in the type1 models where Higgs multiplets come from $\phi_{3}$. The upper panel corresponds to the case with $M^{(3)}=\operatorname{diag}(0,1,-1)$ and the lower panel corresponds to $M^{(3)}=\operatorname{diag}(0,1,0)$ or $\operatorname{diag}(0,0,-1)$.

Next, in figure 2 we show the result about the type 2 models where left-handed and right-handed matter fields have the opposite chirality on the first two tori. ${ }^{11}$ What is quite different from the type1 is that all the numbers of Higgs pairs up to 16 are allowed (at least in our searching region). One-pair Higgs models are also possible.

We find many possibilities to construct the models consistent with the MSSM and we obtain more various flavor structures. In particular, nonfactorizable fluxes enable us to construct one-pair Higgs models. We show the details of such typical models in the next.

\subsection{Minimal models}

Here, we study the details of some candidates for the minimal model like the MSSM. In table 4, we show all the patterns of magnetic flux configurations which give one-pair Higgs models included in the type1, where we choose $M_{C}=0$.

Now, let us focus on the typical one (No. 1) shown in table 4. The flux configuration is set as,

$$
\left(M_{C}^{(1)}, M_{L}^{(1)}, M_{R}^{(1)}\right)=(0,-2,-1),
$$

\footnotetext{
${ }^{11}$ We concentrate on only the case in which the right-handed matter fields come from $\phi_{3}$.
} 


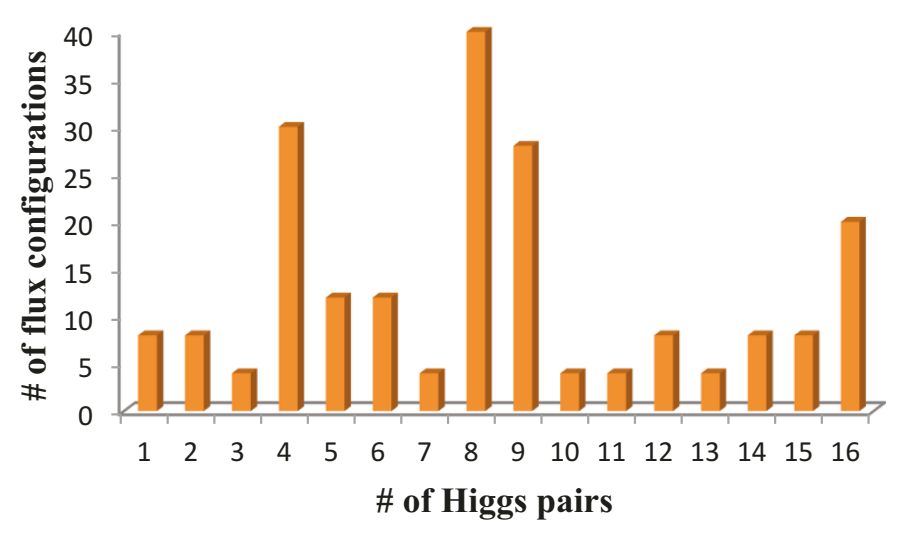

Figure 2. The number of magnetic fluxes for each number of Higgs pairs in the type2 models where Higgs multiplets come from the mixture of $\phi_{1}$ and $\phi_{2}$.

\begin{tabular}{|l|cccccc|cccc|}
\hline & $M_{L}^{(1)}$ & $M_{R}^{(1)}$ & $M_{L}^{(2)}$ & $M_{R}^{(2)}$ & $M_{L}^{(3)}$ & $M_{R}^{(3)}$ & $m_{L}^{(12)}$ & $m_{R}^{(12)}$ & $m_{L}^{(21)}$ & $m_{R}^{(21)}$ \\
\hline No. 1 & -2 & -1 & +1 & +2 & 0 & -1 & +1 & +1 & +1 & +1 \\
\hline No. 2 & -2 & -1 & +1 & +2 & +1 & 0 & +1 & +1 & +1 & +1 \\
\hline No. 3 & +1 & +2 & -2 & -1 & 0 & -1 & +1 & +1 & +1 & +1 \\
\hline No. 4 & +1 & +2 & -2 & -1 & +1 & 0 & +1 & +1 & +1 & +1 \\
\hline No. 5 & -2 & +3 & +1 & +2 & 0 & -1 & +1 & +3 & +1 & +3 \\
\hline No. 6 & +1 & +2 & -2 & +3 & 0 & -1 & +1 & +3 & +1 & +3 \\
\hline No. 7 & -3 & +2 & -2 & -1 & +1 & 0 & +3 & +1 & +3 & +1 \\
\hline No. 8 & -2 & -1 & -3 & +2 & +1 & 0 & +3 & +1 & +3 & +1 \\
\hline
\end{tabular}

Table 4. One-pair Higgs models in type1.

$$
\begin{aligned}
\left(M_{C}^{(2)}, M_{L}^{(2)}, M_{R}^{(2)}\right) & =(0,+1,+2), \\
\left(M_{C}^{(3)}, M_{L}^{(3)}, M_{R}^{(3)}\right) & =(0,0,-1), \\
\left(M_{C}^{(12)}, M_{L}^{(12)}, M_{R}^{(12)}\right) & =(0,+1,+1), \\
\left(M_{C}^{(21)}, M_{L}^{(21)}, M_{R}^{(21)}\right) & =(0,+1,+1) .
\end{aligned}
$$

Then, the differences of fluxes which appear in zero-mode equations of multiplets are obtained as

$$
\begin{aligned}
\mathcal{M}_{L} & =\left(\begin{array}{l}
+2-1 \\
-1-1
\end{array}\right), & \mathcal{M}_{R} & =\left(\begin{array}{l}
-1+1 \\
+1+2
\end{array}\right), \\
M_{C}^{(3)}-M_{L}^{(3)} & =0, & M_{R}^{(3)}-M_{C}^{(3)} & =-1,
\end{aligned}
$$

where a subscript $L$ in $\mathcal{M}_{L}$ means left-handed matter fields, $R$ does right-handed matter and $H$ does Higgs fields. 
With these fluxes, we have the following zero-mode contents, block off-diagonal parts of $\phi_{1}$ and $\phi_{2}$, which are mixed up as shown in eq. (4.8):

$$
\Phi^{\mathcal{I}_{a b}}=\left(\begin{array}{cc|c|cc} 
& Q^{I} & 0 & 0 \\
& L^{I} & 0 & 0 \\
\hline \Xi_{L C} & \Xi_{L C^{\prime}} & & 0 & 0 \\
\hline U^{J} & N^{J} & 0 & \\
D^{J} & E^{J} & 0 &
\end{array}\right)
$$

block diagonal parts of $\phi_{1}$ and $\phi_{2}$, which do not feel the fluxes and each of zero modes exists separately:

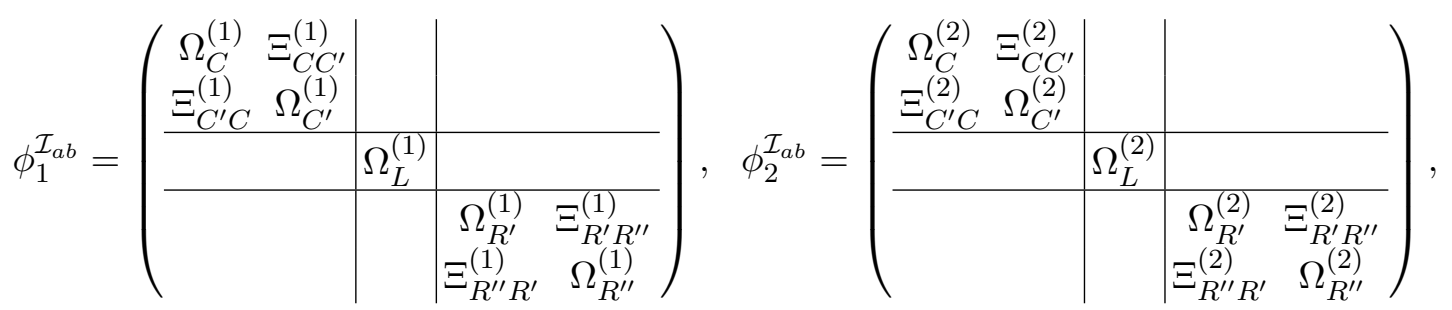

and the fields in $\phi_{3}$ :

$$
\phi_{3}^{\mathcal{I}_{a b}}=\left(\begin{array}{cc|c|cc}
\Omega_{C}^{(3)} & \Xi_{C C^{\prime}}^{(3)} & 0 & 0 & 0 \\
\Xi_{C^{\prime} C}^{(3)} & \Omega_{C^{\prime}}^{(3)} & 0 & 0 & 0 \\
\hline 0 & 0 & \Omega_{L}^{(3)} & H_{u} & H_{d} \\
\hline 0 & 0 & 0 & \Omega_{R^{\prime}}^{(3)} & \Xi_{R^{\prime} R^{\prime \prime}}^{(3)} \\
0 & 0 & 0 & \Xi_{R^{\prime \prime} R^{\prime}}^{(3)} & \Omega_{R^{\prime \prime}}^{(3)}
\end{array}\right)
$$

where we use the same notations as we use in the previous section, however, Higgs doublets do not have a generation index. The Higgs sector feels only factorizable fluxes, introduced in the previous section, $(-1,-1,+1)$ on three tori. The Higgs multiplets appear in $\phi_{3}$ and their degeneracy is given by eq. (3.5), one-pair Higgs. The anti-generations, which have charges opposite to $H_{u}$ and $H_{d}$, do not appear in the conjugate sector, because there is no field having $6 \mathrm{D}$ chirality $(+1,+1,-1) .{ }^{12}$ As for the left-handed sector, $\operatorname{det} \mathcal{M}_{L}=-3$ gives the three generation structure. Since one of the eigenvalues of $\mathcal{M}_{L}$ is negative, they appear in only $\Phi$ with a certain value of the mixing parameter $q_{a b}$, but not in $\phi_{3}$. The value of $q_{a b}$ is determined by the second and the third one of eq. (4.4). The second one always allows two values and one of the two values can satisfy the third condition.

Then, their anti-generations feel the fluxes $-\mathcal{M}_{L}$ on the two tori. Note that, antigenerations in the conjugate sector have charges and fluxes opposite to the corresponding MSSM fields. The minus sign changes only the third condition but not the second one in eq. (4.4). They give a value of the mixing parameter $q_{b a}$ of the conjugate sector. That is, the other one of the two values allowed by the unchanged second condition can satisfy the

\footnotetext{
${ }^{12}$ Without nonfactorizable fluxes, $\phi_{1}$ and $\phi_{2}$ are not mixed up in the Higgs sector. However, they all are eliminated and we simply show the result.
} 


\begin{tabular}{|l|cccccc|cccc|}
\hline & $M_{L}^{(1)}$ & $M_{R}^{(1)}$ & $M_{L}^{(2)}$ & $M_{R}^{(2)}$ & $M_{L}^{(3)}$ & $M_{R}^{(3)}$ & $m_{L}^{(12)}$ & $m_{R}^{(12)}$ & $m_{L}^{(21)}$ & $m_{R}^{(21)}$ \\
\hline No. 1 & -1 & -1 & +2 & -7 & 0 & +1 & -1 & -2 & -1 & -2 \\
\hline No. 2 & 2 & -7 & -1 & -1 & 0 & +1 & -1 & -2 & -1 & -2 \\
\hline No. 3 & -1 & -4 & +2 & -3 & 0 & +1 & +1 & -3 & +1 & -3 \\
\hline No. 4 & 2 & -3 & -1 & -4 & 0 & +1 & +1 & -3 & +1 & -3 \\
\hline No. 5 & -2 & -3 & -9 & -8 & +1 & +1 & +3 & +3 & +7 & +7 \\
\hline No. 6 & -2 & -3 & -9 & -8 & +1 & +1 & +7 & +7 & +3 & +3 \\
\hline No. 7 & -2 & -3 & -9 & -8 & +1 & +1 & -3 & -3 & -7 & -7 \\
\hline No. 8 & -2 & -3 & -9 & -8 & +1 & +1 & -7 & -7 & -3 & -3 \\
\hline
\end{tabular}

Table 5. One-pair Higgs models in type2.

third condition with an additional minus sign. ${ }^{13}$ Thus, three anti-generations also remain. In this case, there is no flux (for the left-handed and the conjugate sector) on the third $T^{2}$ and both of three generation left-handed matter fields and their anti-generations remain finally. We obtain vector-like left-handed matter fields. In contrast, the right-handed sector have nonvanishing fluxes, $M_{C R}^{(3)}=-1$, and anti-generations are eliminated on the $T^{2}$. Finally, chiral right-handed matter fields can be obtained. In all the type1 models with one-pair Higgs multiplets, either the left-handed fields or the right handed fields are vector-like matter fields with their anti-generations.

Next, we study the details of one-pair Higgs models included in the type2 models. There are eight flux configurations, which give one-pair Higgs type2 models, and these are shown in table 5 .

In the first four models, No.1-4, the remaining zero-mode contents are the same as the type1 models. They contains vector-like matter fields in either the left-handed or the right-handed sector (and their conjugate sector).

In contrast, the other four models are attractive. The four models, No. 5-8 in table 5 , are quite similar. Indeed, the four have the same zero-mode contents. That is as follows, block off-diagonal parts of $\phi_{1}$ and $\phi_{2}$, which are mixed up as shown in eq. (4.8):

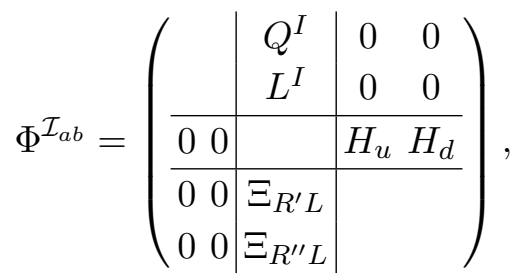

block diagonal parts of $\phi_{1}$ and $\phi_{2}$, which do not feel the fluxes and each of zero modes

\footnotetext{
${ }^{13}$ The second and the third Riemann conditions always give certain values for the mixing parameters of one sector and their conjugate sector simultaneously, at least within the situations considered in this paper.
} 
exists separately:

$$
\phi_{1}^{\mathcal{I}_{a b}}=\left(\begin{array}{c|c|cc|c|c}
\Omega_{C}^{(1)} & \Xi_{C C^{\prime}}^{(1)} & & & \\
\Xi_{C^{\prime} C}^{(1)} & \Omega_{C^{\prime}}^{(1)} & & & \\
\hline & \Omega_{L}^{(1)} & & \\
\hline & & \Omega_{R^{\prime}}^{(1)} & \Xi_{R^{\prime} R^{\prime \prime}}^{(1)} \\
& & \Xi_{R^{\prime \prime} R^{\prime}}^{(1)} & \Omega_{R^{\prime \prime}}^{(1)}
\end{array}\right), \quad \phi_{2}^{\mathcal{I}_{a b}}=\left(\begin{array}{cc|c|c|c}
\Omega_{C}^{(2)} & \Xi_{C C^{\prime}}^{(2)} & & & \\
\hline & \Xi_{C^{\prime} C}^{(2)} & \Omega_{C^{\prime}}^{(2)} & & \\
\hline & \Omega_{L}^{(2)} & & \\
\hline & & & \Omega_{R^{\prime}}^{(2)} & \Xi_{R^{\prime} R^{\prime \prime}}^{(2)} \\
& & & \Xi_{R^{\prime \prime} R^{\prime}}^{(2)} & \Omega_{R^{\prime \prime}}^{(2)}
\end{array}\right),
$$

and the fields in $\phi_{3}$ :

$$
\phi_{3}^{\mathcal{I}_{a b}}=\left(\begin{array}{cc|c|cc}
\Omega_{C}^{(3)} & \Xi_{C C^{\prime}}^{(3)} & 0 & 0 & 0 \\
\Xi_{C^{\prime} C}^{(3)} & \Omega_{C^{\prime}}^{(3)} & 0 & 0 & 0 \\
\hline 0 & 0 & \Omega_{L}^{(3)} & 0 & 0 \\
\hline U^{J} & N^{J} & 0 & \Omega_{R^{\prime}}^{(3)} & \Xi_{R^{\prime} R^{\prime \prime}}^{(3)} \\
D^{J} & E^{J} & 0 & \Xi_{R^{\prime \prime} R^{\prime}}^{(3)} & \Omega_{R^{\prime \prime}}^{(3)}
\end{array}\right)
$$

The chiral matter fields remain just like the MSSM, that is, the models have the chiral matters in the left-handed and the right-handed sector simultaneously because the magnetic fluxes eliminate their anti-generations. Then, they have the MSSM Higgs multiplets correctly in $\Phi$ and also have representations conjugate to the Higgs pair but can not couple with quarks and leptons, that is, inert Higgs pair. In the models, the inert Higgs fields have a parity opposite to the MSSM under a remaining $Z_{2}$ symmetry. That forbids the unusual Yukawa couplings mixing the inert Higgs fields with the MSSM chiral matters.

The extra Higgs multiplets would have SUSY or SUSY breaking mass terms as well as the MSSM Higgs fields. It is consistent with the experimental results if they are heavy to some extent. It is interesting to study various phenomenological aspects of this Higgs sector. We would study them elsewhere.

We show the explicit form of Yukawa matrices of this model in appendix B. According to that, Yukawa matrices can be full-rank and hierarchical, and all the elements of Yukawa matrices can take nonzero values different from each other. Thus, it would be possible to give a realistic pattern of quark and lepton masses and their mixings. ${ }^{14}$

Nonvanishing fluxes for all the sectors are required on the third $T^{2}$ to eliminate all antigeneration appearing in the conjugate sectors of the MSSM contents. For the purpose, we know the unique flux configuration on the third $T^{2}, M^{(3)}=\operatorname{diag}(0,1,-1)$. Such cases are contained in the upper panel of figure 1 . The figure shows there are two flux configurations with $M^{(3)}=\operatorname{diag}(0,1,-1)$ which give two pair of Higgs multiplets. In the two models, there is no anti-generation in the conjugate sectors of any MSSM contents. The models realize the chiral matter fields in exchange for one extra Higgs pair as well as the minimal type 2 models, No. $5-8$ in table 5 (The extra Higgs pair of the type1 models and that of the type2 have opposite charges.).

Let us look back the minimal factorizable models shown in the previous section. The zero-mode contents generated by only magnetic fluxes contain inevitably the six generation

\footnotetext{
${ }^{14}$ In all the one-pair Higgs models, Yukawa matrices have a possibility to give a realistic pattern.
} 
Higgs multiplets, and the remaining anti-generations in two conjugate sectors besides. (Two of the left, right and Higgs sectors remain as the vector-like field.) In contrast, the minimal nonfactorizable flux models have only one Higgs pair as the extra fields, except for the block diagonal parts which are not eliminated by any of factorizable and nonfactorizable fluxes. Furthermore, the extra Higgs fields are supposed to have mass terms. This way, we see that the nonfactorizable flux is capable to derive the realistic model from the 10D magnetized SYM theory.

\subsection{Nonfactorizable flux models on $T^{6} / Z_{2}$ orbifolds}

There are other possibilities for model building. We can consider nonfactorizable fluxes with orbifold projection, where magnetic fluxes have to be five-block forms because (continuous) Wilson-lines cannot be introduced to break the gauge group by the orbifold projection. When we consider the $Z_{2}$ projection eq. (3.13) either even or odd modes of zero-modes remain. How to derive even and odd functions from eqs. (3.6) and (3.7) has already known for factorizable fluxes in ref. [19], and we find that even and odd functions can be derived from eqs. (4.6) and (4.7) as natural generalization of the factorizable case eq. (3.12) as,

$$
\begin{aligned}
\phi_{\text {even }}^{\vec{j}}(\vec{z}) & =\frac{1}{\sqrt{2}}\left(\phi^{\vec{j}, \mathcal{M}}(\vec{z})+\phi^{\vec{e}-\vec{j}, \mathcal{M}}(\vec{z})\right), \\
\phi_{\text {odd }}^{\vec{j}}(\vec{z}) & =\frac{1}{\sqrt{2}}\left(\phi^{\vec{j}, \mathcal{M}}(\vec{z})-\phi^{\vec{e}-\vec{j}, \mathcal{M}}(\vec{z})\right),
\end{aligned}
$$

where,

$$
\vec{e} \equiv \vec{e}_{1}+\vec{e}_{2}=\left(\begin{array}{l}
1 \\
0
\end{array}\right)+\left(\begin{array}{l}
0 \\
1
\end{array}\right)=\left(\begin{array}{l}
1 \\
1
\end{array}\right) .
$$

Note that we use the following relations to obtain these functions,

$$
\phi^{\vec{j}, \mathcal{M}}(-\vec{z})=\phi^{\vec{e}-\vec{j}, \mathcal{M}}(\vec{z}) .
$$

According to that, we find that a rule for the number of the degenerate zero-modes after a $Z_{2}$ projection is the same as factorizable cases and we see the rule in table 3 with replacing $M$ by $\operatorname{det} \mathcal{M} \cdot{ }^{15}$

On such a background, we search flux configurations to give the three generation models in a systematic way and the range between -10 and 10. As a result, we could not construct three generation models with four-block or five-block fluxes because SUSY conditions are severe.

\section{Conclusions and discussions}

We have carried out a systematic search for possibilities to construct plausible models focusing on only the visible sector, where we obtain preserved $\mathcal{N}=1$ SUSY, the SM gauge

\footnotetext{
${ }^{15}$ Strictly speaking, there are some exceptional case, however, it has nothing to do with us as far as we aim to the three generation models.
} 
group, all the MSSM fields, three generation structures of quarks and leptons and full-rank Yukawa matrices.

First, we have done that considering only the factorizable fluxes, then we find that the Pati-Salam models with six pairs of Higgs multiplets can be constructed.

An orbifold projection could eliminate exotic modes without changing three generation structures of quarks and leptons. It also can change the number of degenerate zero-modes if $Z_{2}$ act on the torus where flavor structures are generated, and we try to use that to generate three generation structures other than previous ones. With such an orbifold projection, (continuous) Wilson-lines are not allowed and we have to break the gauge group using only magnetic fluxes. Thus, we have to consider five-block magnetic fluxes for which SUSY conditions become more severe and we find it impossible.

We can consider nonfactorizable fluxes which cross over two tori. Considering nonfactorizable fluxes, more various model buildings become possible. With such fluxes we tried to construct the Pati-Salam models, and we find that there are infinite possibilities to give three generation models as a result of systematic research. We also find some particular values are allowed for the number of Higgs pairs if Higgs multiplets come from $\phi_{3}$, in contrast any numbers of Higgs pairs can be realized in the other case. Furthermore, the quite attractive fact is that we can realize the $4 \mathrm{D}$ chiral spectra and the Higgs sector of the MSSM with one extra Higgs pair by using only magnetic fluxes. Finally, we tried to construct nonfactorizable flux models on $T^{6} / Z_{2}$ orbifolds. As a result we cannot find a flux configuration to give us the plausible model.

In this paper we have found many possibilities to give the plausible models, which have the three generation quark and lepton multiplets and full-rank Yukawa matrices, other than the one studied in ref. [15]. Plausible flux configurations give only the PatiSalam models, where we need to introduce Wilson-lines to break the gauge group. In the Pati-Salam models with only factorizable fluxes, Yukawa coupling possesses the flavor symmetry $\Delta(27)[9]$. It is interesting to study how such structures would be changed by nonfactorizable fluxes. Furthermore, introducing nonfactorizable fluxes has a close connection with the moduli stabilization. We will study these features of nonfactorizable fluxes elsewhere.

The five-block flux models not needing Wilson-lines cannot be realized in 10D magnetized SYM theory. However, our study can be extended straightforwardly to SYM theories in a lower than ten-dimensional spacetime, or even to the mixture of SYM theories with a different dimensionality. In such theories, we might be able to build the five-block and more various models.

As other generalizations, we can consider generalized torus in which off-diagonal elements of general complex structure $\Omega$ have nonzero values, or more complicated manifold, and that would give us a new flavor landscape.

Although we have studied 10D SYM as our starting point, it is important to embed our models in superstring theories, i.e. magnetized D-brane models $[1-4,6]$. In such embedding, string consistency constrains model building and also inclusion of orientifold planes leads to new types of matter fields. Such a study is very interesting, but it is beyond our scope of this paper. 
Finally, our models derived from SYM theory might be embedded in the matrix models $[29,30]$. In that case, it would give us a new motivation to study the magnetized SYM theory.

\section{Acknowledgments}

H.A. was supported in part by the Grant-in-Aid for Scientific Research No. 25800158 from the Ministry of Education, Culture, Sports, Science and Technology (MEXT) in Japan. T.K. was supported in part by the Grant-in-Aid for Scientific Research No. 25400252 from the MEXT in Japan. H.O. was supported in part by the JSPS Grant-in-Aid for Scientific Research (S) No. 22224003, for young Scientists (B) No. 25800139 from the MEXT in Japan. K.S. was supported in part by a Grant-in-Aid for JSPS Fellows No. 25.4968 and a Grant for Excellent Graduate Schools from the MEXT in Japan. Y.T. was supported in part by a Grant for Excellent Graduate Schools from the MEXT in Japan. Y.T. would like to thank T. Shohji and K. Oh for a correspondence.

\section{A Exotic modes with the pattern1 fluxes}

In this section, we show how the $Z_{2}$-projection work for the matter profile (3.11) caused by the type 1 magnetic fluxes in table 1 . We consider $Z_{2}$-projection introduced in section 3 which acts on the second and the third tori with the following projection operator,

$$
P_{a b}=\left(\begin{array}{ccc}
-\mathbf{1}_{4} & 0 & 0 \\
0 & +\mathbf{1}_{2} & 0 \\
0 & 0 & +\mathbf{1}_{2}
\end{array}\right)
$$

This projection removes most of the massless exotic modes $\Xi_{a b}^{(r)}$ and massless diagonal components $\Omega_{a}^{(r)}$. As a result, the matter contents on the orbifold $T^{6} / Z_{2}$ are found as

$$
\begin{aligned}
\phi_{1}^{\mathcal{I}_{a b}}= & \left(\begin{array}{cc|c|cc}
\Omega_{C}^{(1)} & \Xi_{C C^{\prime}}^{(1)} & 0 & 0 & 0 \\
\Xi_{C^{\prime} C}^{(1)} & \Omega_{C^{\prime}}^{(1)} & 0 & 0 & 0 \\
\hline 0 & 0 & \Omega_{L}^{(1)} & H_{u}^{K} & H_{d}^{K} \\
\hline 0 & 0 & 0 & \Omega_{R^{\prime}}^{(1)} & \Xi_{R^{\prime} R^{\prime \prime}}^{(1)} \\
\hline 0 & 0 & 0 & \Xi_{R^{\prime \prime} R^{\prime}}^{(1)} & \Omega_{R^{\prime \prime}}^{(1)}
\end{array}\right), \\
\phi_{2}^{\mathcal{I}_{a b}}= & \left(\begin{array}{cc|c|cc}
0 & 0 & Q^{I} & 0 & 0 \\
0 & 0 & L^{I} & 0 & 0 \\
\hline 0 & 0 & 0 & 0 & 0 \\
\hline 0 & 0 & 0 & 0 & 0 \\
0 & 0 & 0 & 0 & 0
\end{array}\right), \quad \phi_{3}^{\mathcal{I}_{a b}}=\left(\begin{array}{cc|c|cc}
0 & 0 & 0 & 0 & 0 \\
0 & 0 & 0 & 0 & 0 \\
\hline 0 & 0 & 0 & 0 & 0 \\
\hline U^{J} & N^{J} & 0 & 0 & 0 \\
D^{J} & E^{J} & 0 & 0 & 0
\end{array}\right),
\end{aligned}
$$

where $I, J=1,2,3$ and $K=1, \ldots, 6$ label the generations as in eq. (3.11). Some massless exotic modes $\Xi_{a b}^{(1)}$ and open string moduli $\Omega_{a}^{(1)}$ still remain, and that is one of the open problems in the $T^{6} / Z_{2}$ magnetized orbifold model. Here, we can only assume these exotic modes to become massive, as well as in ref. [15], through some nonperturbative effects [27, $28]$ or higher-order corrections, so that they decouple from the low-energy physics. 


\section{B Yukawa matrices in a one-pair Higgs model}

We show an explicit form of Yukawa matrices in a one-pair Higgs model. Yukawa couplings of No.5 model in type 2 are written by

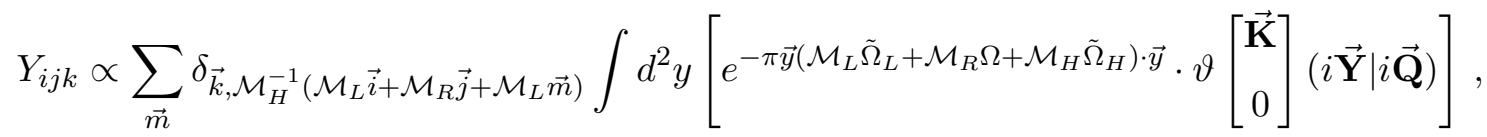

where

$$
\begin{aligned}
\tilde{\Omega}_{L} & =\hat{\Omega}_{L} \cdot \Omega, \quad \tilde{\Omega}_{H}=\hat{\Omega}_{H} \cdot \Omega \\
\overrightarrow{\mathbf{K}} & \equiv\left(\begin{array}{c}
\vec{k} \\
(\vec{i}-\vec{j}+\vec{m}) \frac{\mathcal{M}_{L}\left(\mathcal{M}_{L}+\mathcal{M}_{R}\right)^{-1} \mathcal{M}_{R}}{\operatorname{det} \mathcal{M}_{L} \operatorname{det} \mathcal{M}_{R}}
\end{array}\right) \\
\overrightarrow{\mathbf{Y}} & \equiv\left(\begin{array}{c}
\left(\mathcal{M}_{L} \tilde{\Omega}_{L}+\mathcal{M}_{R} \Omega+\mathcal{M}_{H} \tilde{\Omega}_{H}\right) \cdot \vec{y} \\
\left.\left(\operatorname{det} \mathcal{M}_{L} \operatorname{det} \mathcal{M}_{R}\right)\left(\mathcal{M}_{L} \tilde{\Omega}_{L}\left(\mathcal{M}_{L}^{-1}\right)^{T}-\mathcal{M}_{R} \Omega\left(\mathcal{M}_{R}^{-1}\right)^{T}\right) \cdot \vec{y}\right)
\end{array}\right) \\
\overrightarrow{\mathbf{Q}} & \equiv\left(\begin{array}{cr}
\mathcal{M}_{L} \tilde{\Omega}_{L}+\mathcal{M}_{R} \Omega+\mathcal{M}_{H} \tilde{\Omega}_{H} & \left(\operatorname{det} \mathcal{M}_{L} \operatorname{det} \mathcal{M}_{R}\right)\left(\mathcal{M}_{L} \tilde{\Omega}_{L} \cdot\left(\mathcal{M}_{L}^{-1}\right)^{T}-\mathcal{M}_{R} \Omega \cdot\left(\mathcal{M}_{R}^{-1}\right)^{T}\right) \\
\left(\operatorname{det} \mathcal{M}_{L} \operatorname{det} \mathcal{M}_{R}\right)\left(\tilde{\Omega}_{L}-\Omega\right) & \left(\operatorname{det} \mathcal{M}_{L} \operatorname{det} \mathcal{M}_{R}\right)^{2}\left(\tilde{\Omega}_{L} \mathcal{M}_{L}^{-1}+\Omega \mathcal{M}_{R}^{-1}\right)
\end{array}\right) .
\end{aligned}
$$

We define

$$
\eta\left[(\vec{i}-\vec{j}+\vec{m}) \frac{\mathcal{M}_{L}\left(\mathcal{M}_{L}+\mathcal{M}_{R}\right)^{-1} \mathcal{M}_{R}}{\operatorname{det} \mathcal{M}_{L} \operatorname{det} \mathcal{M}_{R}}\right] \equiv \vartheta\left[\begin{array}{c}
\overrightarrow{\mathbf{K}} \\
0
\end{array}\right](i \overrightarrow{\mathbf{Y}} \mid i \overrightarrow{\mathbf{Q}}),
$$

and then $(1,1)$ element can be written using $\eta$;

$$
\lambda_{11}=\eta[(0,0)]+\eta\left[\left(0, \frac{1}{3}\right)\right]+\eta\left[\left(0, \frac{2}{3}\right)\right]+\eta\left[\left(\frac{1}{3}, 0\right)\right] .
$$

Similarly, other elements are written by

$$
\begin{aligned}
& \lambda_{12}=\eta\left[\left(\frac{4}{9}, \frac{5}{9}\right)\right]+\eta\left[\left(\frac{4}{9}, \frac{8}{9}\right)\right]+\eta\left[\left(\frac{4}{9}, \frac{2}{9}\right)\right]+\eta\left[\left(\frac{7}{9}, \frac{5}{9}\right)\right], \\
& \lambda_{13}=\eta\left[\left(\frac{8}{9}, \frac{1}{9}\right)\right]+\eta\left[\left(\frac{8}{9}, \frac{4}{9}\right)\right]+\eta\left[\left(\frac{8}{9}, \frac{7}{9}\right)\right]+\eta\left[\left(\frac{2}{9}, \frac{1}{9}\right)\right], \\
& \lambda_{21}=\eta\left[\left(\frac{2}{9}, \frac{2}{9}\right)\right]+\eta\left[\left(\frac{2}{9}, \frac{5}{9}\right)\right]+\eta\left[\left(\frac{2}{9}, \frac{8}{9}\right)\right]+\eta\left[\left(\frac{5}{9}, \frac{2}{9}\right)\right], \\
& \lambda_{22}=\eta\left[\left(\frac{2}{3}, \frac{7}{9}\right)\right]+\eta\left[\left(\frac{2}{3}, \frac{1}{9}\right)\right]+\eta\left[\left(\frac{2}{3}, \frac{4}{9}\right)\right]+\eta\left[\left(0, \frac{7}{9}\right)\right], \\
& \lambda_{23}=\eta\left[\left(\frac{1}{9}, \frac{1}{3}\right)\right]+\eta\left[\left(\frac{1}{9}, \frac{2}{3}\right)\right]+\eta\left[\left(\frac{1}{9}, 0\right)\right]+\eta\left[\left(\frac{4}{9}, \frac{1}{3}\right)\right], \\
& \lambda_{31}=\eta\left[\left(\frac{4}{9}, \frac{7}{9}\right)\right]+\eta\left[\left(\frac{4}{9}, \frac{1}{9}\right)\right]+\eta\left[\left(\frac{4}{9}, \frac{4}{9}\right)\right]+\eta\left[\left(\frac{7}{9}, \frac{7}{9}\right)\right], \\
& \lambda_{32}=\eta\left[\left(\frac{8}{9}, \frac{1}{3}\right)\right]+\eta\left[\left(\frac{8}{9}, \frac{2}{3}\right)\right]+\eta\left[\left(\frac{8}{9}, 0\right)\right]+\eta\left[\left(\frac{2}{9}, \frac{1}{3}\right)\right], \\
& \lambda_{33}=\eta\left[\left(\frac{1}{3}, \frac{8}{9}\right)\right]+\eta\left[\left(\frac{1}{3}, \frac{2}{9}\right)\right]+\eta\left[\left(\frac{1}{3}, \frac{5}{9}\right)\right]+\eta\left[\left(\frac{2}{3}, \frac{8}{9}\right)\right],
\end{aligned}
$$


and total Yukawa matrices can be written as follows

$$
Y_{i j k} \propto \int d^{2} y\left[e^{-\pi \vec{y}\left(\mathcal{M}_{L} \tilde{\Omega}_{L}+\mathcal{M}_{R} \tilde{\Omega}_{R}+\mathcal{M}_{H} \Omega\right) \vec{y}}\left(\begin{array}{ccc}
\lambda_{11} & \lambda_{12} & \lambda_{13} \\
\lambda_{21} & \lambda_{22} & \lambda_{23} \\
\lambda_{31} & \lambda_{32} & \lambda_{33}
\end{array}\right)\right] .
$$

The values of elements are different from each other, and we cannot find any non-Abelian flavor symmetry there.

Open Access. This article is distributed under the terms of the Creative Commons Attribution License (CC-BY 4.0), which permits any use, distribution and reproduction in any medium, provided the original author(s) and source are credited.

\section{References}

[1] C. Bachas, A Way to break supersymmetry, hep-th/9503030 [INSPIRE].

[2] R. Blumenhagen, L. Görlich, B. Körs and D. Lüst, Noncommutative compactifications of type-I strings on tori with magnetic background flux, JHEP 10 (2000) 006 [hep-th/0007024] [INSPIRE].

[3] C. Angelantonj, I. Antoniadis, E. Dudas and A. Sagnotti, Type I strings on magnetized orbifolds and brane transmutation, Phys. Lett. B 489 (2000) 223 [hep-th/0007090] [INSPIRE].

[4] R. Blumenhagen, B. Körs and D. Lüst, Type I strings with F flux and B flux, JHEP 02 (2001) 030 [hep-th/0012156] [INSPIRE].

[5] D. Cremades, L.E. Ibáñez and F. Marchesano, Computing Yukawa couplings from magnetized extra dimensions, JHEP 05 (2004) 079 [hep-th/0404229] [INSPIRE].

[6] L.E. Ibanez and A.M. Uranga, String theory and particle physics: An introduction to string phenomenology, University Press, Cambridge, U.K. (2012), pg. 673.

[7] H. Abe, K.-S. Choi, T. Kobayashi and H. Ohki, Three generation magnetized orbifold models, Nucl. Phys. B 814 (2009) 265 [arXiv:0812.3534] [INSPIRE].

[8] H. Abe, K.-S. Choi, T. Kobayashi and H. Ohki, Higher Order Couplings in Magnetized Brane Models, JHEP 06 (2009) 080 [arXiv: 0903.3800] [InSPIRE].

[9] H. Abe, K.-S. Choi, T. Kobayashi and H. Ohki, Non-Abelian Discrete Flavor Symmetries from Magnetized/Intersecting Brane Models, Nucl. Phys. B 820 (2009) 317 [arXiv: 0904.2631] [INSPIRE].

[10] H. Abe, K.-S. Choi, T. Kobayashi and H. Ohki, Magnetic flux, Wilson line and orbifold, Phys. Rev. D 80 (2009) 126006 [arXiv:0907.5274] [INSPIRE].

[11] H. Abe, K.-S. Choi, T. Kobayashi and H. Ohki, Flavor structure from magnetic fluxes and non-Abelian Wilson lines, Phys. Rev. D 81 (2010) 126003 [arXiv:1001.1788] [INSPIRE].

[12] M. Berasaluce-Gonzalez, P.G. Camara, F. Marchesano, D. Regalado and A.M. Uranga, Non-Abelian discrete gauge symmetries in 4d string models, JHEP 09 (2012) 059 [arXiv: 1206.2383] [INSPIRE].

[13] F. Marchesano, D. Regalado and L. Vazquez-Mercado, Discrete flavor symmetries in D-brane models, JHEP 09 (2013) 028 [arXiv:1306.1284] [INSPIRE]. 
[14] H. Abe, T. Kobayashi, H. Ohki and K. Sumita, Superfield description of 10D SYM theory with magnetized extra dimensions, Nucl. Phys. B 863 (2012) 1 [arXiv: 1204.5327] [InSPIRE].

[15] H. Abe, T. Kobayashi, H. Ohki, A. Oikawa and K. Sumita, Phenomenological aspects of $10 D$ SYM theory with magnetized extra dimensions, Nucl. Phys. B 870 (2013) 30 [arXiv: 1211.4317] [INSPIRE].

[16] I. Antoniadis, A. Kumar and B. Panda, Fermion Wavefunctions in Magnetized branes: Theta identities and Yukawa couplings, Nucl. Phys. B 823 (2009) 116 [arXiv:0904.0910] [INSPIRE].

[17] L. De Angelis, R. Marotta, F. Pezzella and R. Troise, More About Branes on a General Magnetized Torus, JHEP 10 (2012) 052 [arXiv:1206.3401] [INSPIRE].

[18] M. Sakamoto and S. Tanimura, An Extension of Fourier analysis for the $n$ torus in the magnetic field and its application to spectral analysis of the magnetic Laplacian, J. Math. Phys. 44 (2003) 5042 [hep-th/0306006] [INSPIRE].

[19] H. Abe, T. Kobayashi and H. Ohki, Magnetized orbifold models, JHEP 09 (2008) 043 [arXiv:0806.4748] [INSPIRE].

[20] S. Groot Nibbelink and P.K.S. Vaudrevange, Schoen manifold with line bundles as resolved magnetized orbifolds, JHEP 03 (2013) 142 [arXiv: 1212.4033] [INSPIRE].

[21] Y. Fujimoto, T. Kobayashi, T. Miura, K. Nishiwaki and M. Sakamoto, Shifted orbifold models with magnetic flux, Phys. Rev. D 87 (2013) 086001 [arXiv:1302.5768] [INSPIRE].

[22] N. Marcus, A. Sagnotti and W. Siegel, Ten-dimensional Supersymmetric Yang-Mills Theory in Terms of Four-dimensional Superfields, Nucl. Phys. B 224 (1983) 159 [INSPIRE].

[23] N. Arkani-Hamed, T. Gregoire and J.G. Wacker, Higher dimensional supersymmetry in $4 D$ superspace, JHEP 03 (2002) 055 [hep-th/0101233] [INSPIRE].

[24] K.-S. Choi, T. Kobayashi, R. Maruyama, M. Murata, Y. Nakai et al., E(6,7,8) Magnetized Extra Dimensional Models, Eur. Phys. J. C 67 (2010) 273 [arXiv:0908.0395] [InSPIRE].

[25] T. Kobayashi, R. Maruyama, M. Murata, H. Ohki and M. Sakai, Three-generation Models from $E_{8}$ Magnetized Extra Dimensional Theory, JHEP 05 (2010) 050 [arXiv:1002.2828] [INSPIRE].

[26] Y. Hamada and T. Kobayashi, Massive Modes in Magnetized Brane Models, Prog. Theor. Phys. 128 (2012) 903 [arXiv: 1207.6867] [INSPIRE].

[27] L.E. Ibáñez and A.M. Uranga, Neutrino Majorana Masses from String Theory Instanton Effects, JHEP 03 (2007) 052 [hep-th/0609213] [INSPIRE].

[28] M. Cvetič, R. Richter and T. Weigand, Computation of D-brane instanton induced superpotential couplings: Majorana masses from string theory, Phys. Rev. D 76 (2007) 086002 [hep-th/0703028] [INSPIRE].

[29] J. Nishimura and A. Tsuchiya, Realizing chiral fermions in the type IIB matrix model at finite N, JHEP 12 (2013) 002 [arXiv:1305.5547] [INSPIRE].

[30] H. Aoki, Probability distribution over some phenomenological models in the matrix model compactified on a torus, Prog. Theor. Exp. Phys. (2013) 093B04 [arXiv:1303.3982] [INSPIRE]. 